\title{
Mechanical Solitaire Thrombectomy with Low-Dose Booster Tirofiban Injection
}

\author{
Duck-Ho Goh, MD', Sung-Chul Jin, MD², Hae Woong Jeong, MD³, Sam Yeol Ha, MD
}

Purpose: Mechanical thrombectomy using a Solitaire stent has been associated with a high recanalization rate and favorable clinical outcome in intra-arterial thrombolysis. To achieve a higher recanalization rate for mechanical Solitaire thrombectomy, we used an intra-arterial low-dose booster tirofiban injection into the occluded segment after stent deployment. We report the safety and recanalization rates for mechanical Solitaire thrombectomy with a low-dose booster tirofiban injection.

Materials and Methods: Between February and March 2013, 13 consecutive patients underwent mechanical Solitaire thrombectomy with low-dose booster tirofiban injection. The occlusion sites included the proximal middle cerebral artery (5 patients), the internal carotid artery (5 patients), the top of the basilar artery ( 2 patients) and the distal middle cerebral artery ( $M 2$ segment, 1 patient). Six patients underwent bridge treatment, including intravenous tissue plasminogen activator. Tirofiban of $250 \mu \mathrm{g}$ was used in all patients except one $(500 \mu \mathrm{g})$. All occluded vessels were recanalized after 3 attempts at stent retrieval ( 1 time, $n=9 ; 2$ times, $n=2 ; 3$ times, $n=2$ ).

Results: Successful recanalization was achieved in all patients $(\mathrm{TICl} 3, n=8 ; \mathrm{TICl} 2 \mathrm{~b}, \mathrm{n}=5)$. Procedural complications developed in 3 patients (subarachnoid hemorrhage, $n=2$; hemorrhagic transformation, $\mathrm{n}=1$ ). Mortality occurred in one patient with a basilar artery occlusion due to reperfusion brain swelling after mechanical Solitaire thrombectomy with low-dose booster tirofiban injection. Favorable clinical outcome ( $\mathrm{mRS} \leq 2)$ was observed in 8 patients $(61.5 \%)$.

Conclusion: Our modified mechanical Solitaire thrombectomy method using a low-dose booster tirofiban injection might enhance the recanalization rate with no additive hemorrhagic complications.

Key Words : Mechanical thrombectomy; Solitaire stent; Tirofiban

\footnotetext{
'Department of Neurosurgery, Hanmaeum Changwon Hospital, Changwon, Korea

Departments of ${ }^{2}$ Neurosurgery and ${ }^{4}$ Neurology, Inje University Haeundae Paik Hospital, Busan, Korea

${ }^{3}$ Department of Radiology, Inje University Busan Paik Hospital, Busan, Korea

Received August 14, 2016; accepted after revision August 22, 2016.

Correspondence to: Sung-Chul Jin, MD, PhD, Department of Neurosurgery, Inje University, Haeundae Paik Hospital, 1435 Jwa-dong Haeundae-gu, Busan 612-043, Korea.

Tel. 82.51.797.0607 Fax. 82.51.703.0434 E-mail: kusmal@hanmail.net

This is an Open Access article distributed under the terms of the Creative Commons Attribution Non-Commercial License (http://creativecommons.org/licenses/by-nc/3.0) which permits unrestricted non-commercial use, distribution, and reproduction in any medium, provided the original work is properly cited.
} 
Mechanical thrombectomy using stent devices has been considered as a standard treatment modality due to its favorable clinical and radiological outcomes in the treatment of acute ischemic stroke caused by intracranial large vessel occlusion $[1,2]$. However, despite the learning curve for the Solitaire stent, our experiences with mechanical thrombectomy using the Solitaire stent have resulted in a lower recanalization rate than previously reported [2-4].

Tirofiban is a relatively short-acting and reversible glycoprotein IIb/IIIa receptor inhibitor and has been increasingly used in the treatment of acute thromboembolism caused by a coiling procedure or salvage treatment of acute ischemic stroke refractory to using conventional techniques $[5,6]$. In a previous trial, we combined tirofiban injection (dosage, $0 \sim 800 \mu \mathrm{g}$ ) with ballooning for the treatment of an acute middle cerebral artery occlusion. No procedure-related hemorrhage or hemorrhagic transformation was observed after intraarterial thrombolysis using low-dose tirofiban injection, consistent with previous reports $[7,8]$.

Unless the incidence of procedure-related hemorrhage increases, we propose that a low-dose booster tirofiban injection will accelerate thrombolysis in the occluded vessel under deployment of a stent, temporarily maintaining antegrade flow of the occluded vessel. We modified the procedure for mechanical Solitaire thrombectomy in the following ways: 1) intraarterial low-dose booster tirofiban $(250 \mu \mathrm{g})$ injection after Solitaire stent was deployed in the occluded vessel; 2) serial control angiograms were conducted to evaluate current blood flow status in the treated occluded vessel using 2-minute intervals; and 3) the deployed stent was retrieved when serial angiograms showed no antegrade flow of the occluded vessel or at 10 minutes after the deployment of the stent in cases of maintained blood flow in the occluded vessels.

We retrospectively evaluated the efficacy and safety of mechanical Solitaire thrombectomy with low-dose booster tirofiban injection.

\section{METHODS}

\section{Patients}

We performed mechanical Solitaire thrombectomy with low-dose booster tirofiban injection in 13 consecutive patients with acute ischemic stroke caused by intracranial large vessel occlusion from February 2013 to March 2013. The study was approved by the Institutional Review Board.

Stroke neurologists performed neurological assess- ments based on the National Institute of Health Stroke Scale (NIHSS) Score. Most patients underwent a nonenhanced brain CT, CT angiography, CT perfusion, and brain MRI. The brain MRI protocol included diffusionweighted imaging, fluid-attenuated inversion recovery sequence, and T2 gradient-recall echo imaging.

Mechanical Solitaire thrombectomy with low-dose booster tirofiban injection was generally performed in patients arriving within 8 hours of symptom onset who had a NIHSS $\geq 4$. Intracranial hemorrhage was ruled out using plain CT in patients with neurological deficits. Subsequently, large vessel occlusion and the status of perfusion/diffusion mismatch were confirmed using CT angiography with perfusion and MR diffusion imaging. Exceptionally, patients with NIHSS $<4$ were considered for treatment in cases of fluctuating or progressive neurological deficits. Patients beyond a time window of 8 hours were considered for treatment in cases of remarkable perfusion/diffusion mismatch lesions, suggesting a relevant proportion of penumbra or a salvage procedure to avoid decompressive craniectomy. Patients with perfusion/diffusion-matched lesions were considered for treatment restrictively within 3 hours of symptom onset and a NIHSS $>10$.

Intravenous recombinant tissue plasminogen activator (dose; $0.6 \mathrm{mg} / \mathrm{kg}$ ) was administered before the procedure as a bridge therapy if patients with acute ischemic attack generally met the inclusion and exclusion criteria of intravenous thrombolysis.

The functional outcome was assessed based on the NIHSS at 1 week after procedure and the modified Rankin scale at 3 months after discharge.

\section{Endovascular treatment technique}

An interventional neurosurgeon, with experience of operating on more than 600 interventional procedures, performed all endovascular treatments under the informed consent of patients or the patients' families. All patients were treated under local anesthesia with sedative agents. A 6F Envoy guiding catheter (Codman, Raynham, MA) coaxially introduced into a $6 \mathrm{~F}$ shuttle catheter (Cook Medical Inc., Bloomington, IN) was placed in the internal carotid or vertebral artery. The guiding catheters were flushed continuously with heparinized saline (5000 U/liter).

In all patients, mechanical thrombectomy was performed using a Solitaire stent of $6 \mathrm{~mm}$ in diameter and $30 \mathrm{~mm}$ in length. An Excelsior xt 27 microcatheter (Stryker, Fremont, California) was navigated distal to the thrombus over a Synchro 0.014 microwire (Stryker, Fremont, California) and positioned approximately 1.5 


\section{Duck-Ho Goh, et al.}

$\mathrm{cm}$ distal to the thrombus under confirmation of contrast injection. The Solitaire stent (ev3 Inc., Irvine, California) was subsequently advanced to the distal tip of the microcatheter and fully deployed across the thrombotic lesion. A control angiogram was used to evaluate the recanalization status of the thrombotic lesion after stent deployment. Intermittent hand injections of intra-arterial tirofiban (typical dose: $250 \mu$ $\mathrm{g}, 50 \mu \mathrm{g}$ at a time) without a loading dose were performed through the delivery microcatheter. Control angiograms were repeatedly performed using 2-minute intervals to assess the progression of the thrombotic lesion across which the stent was deployed. If the control angiograms showed no antegrade flow of the thrombotic lesion or at 10 minutes after stent deployment in cases of maintained blood flow, the partial resheathed Solitaire stent and the delivery microcatheter were removed as a system until the unsheathed Solitaire stent was positioned in the distal tip of the guiding catheter. Thereafter, the partial re-sheathed Solitaire stent, delivery microcatheter, and guiding catheter were withdrawn through the shuttle catheter, while applying no flushing of the heparinized saline into the guiding and shuttle catheters. A control angiogram was performed to assess recanalization after the retrieval of the stent system. The recanalization status was assessed in the final angiogram and classified according to the Thrombolysis in Cerebral Ischemia (TICI) scale.9 Successful recanalization was defined as a TICI grade of $2 \mathrm{~b}$ or 3 .

The entire procedural steps were repeated (a maximum of 3 times) until recanalization was achieved, as defined by successful recanalization (TICI grade $\geq$ $2 \mathrm{~b}$ ), and a maximum dose of $500 \mu \mathrm{g}$ of tirofiban was injected. No subsequent additional procedures were performed.

All patients underwent non-enhanced CT scans immediately after, and 24 hours after the procedure to investigate possible hemorrhagic complications. Hemorrhagic transformations were categorized and described as per European Cooperative Acute Stroke Study criteria [10].

\section{RESULTS}

The admission NIHSS score ranged from 4 to 19 (median; 15) (Table 1). The occlusion sites were internal carotid artery (ICA) $(n=3)$, T-bifurcation of ICA ( $n=2)$, the M1 segment of middle cerebral artery $(n=5)$, the M2 segment of middle cerebral artery $(n=1)$,

Table 1. Baseline Clinical and Procedural Characteristics in 13 Patients Who Underwent Mechanical Solitaire Thrombectomy with Low Dose Booster Injection of Tirofiban

\begin{tabular}{|c|c|c|c|c|c|c|c|c|c|}
\hline $\begin{array}{l}\text { Patient } \\
\text { No. }\end{array}$ & $\begin{array}{c}\text { Age } \\
\text { in years }\end{array}$ & Sex & Occlusion site & IV tPA & $\begin{array}{l}\text { Tirofiban } \\
\text { Dose }(\mu \mathrm{g})\end{array}$ & $\begin{array}{c}\text { Characteristics of } \\
\text { the repeated } \\
\text { angiograms }\end{array}$ & $\begin{array}{l}\text { Frequency of } \\
\text { stent retrieval }\end{array}$ & $\mathrm{TICl}$ & $\begin{array}{c}\text { Procedural } \\
\text { complications }\end{array}$ \\
\hline 1 & 53 & $M$ & $\mathrm{~T}$ & No & 500 & Maintained flow & 1 & 3 & No \\
\hline 2 & 53 & $M$ & Basilar artery & No & 250 & Decreased flow & 1 & $2 b$ & No \\
\hline 3 & 54 & $M$ & M1 & No & 250 & Decreased flow & 2 & 3 & No \\
\hline 4 & 77 & $M$ & M1 & No & 250 & Maintained flow & 1 & 3 & No \\
\hline 5 & 66 & $\mathrm{~F}$ & Basilar artery & No & 250 & Maintained flow & 1 & 3 & No \\
\hline 6 & 59 & $M$ & $\mathrm{~T}$ & Yes & 250 & Decreased flow & 3 & $2 b$ & No \\
\hline 7 & 58 & $M$ & M1 & Yes & 250 & Maintained flow & 1 & 3 & SAH \\
\hline 8 & 49 & $\mathrm{~F}$ & M2 & No & 250 & Maintained flow & 1 & $2 b$ & $\mathrm{SAH}$ \\
\hline 9 & 45 & $\mathrm{~F}$ & M1 & Yes & 250 & Maintained flow & 1 & 3 & No \\
\hline 10 & 80 & $\mathrm{~F}$ & M1 & Yes & 250 & Decreased flow & 1 & 3 & No \\
\hline 11 & 69 & $\mathrm{M}$ & ICA & Yes & 250 & Maintained flow & 1 & 3 & No \\
\hline 12 & 79 & $\mathrm{~F}$ & ICA & No & 250 & Maintained flow * & 3 & $2 b$ & Transformation \\
\hline 13 & 63 & $M$ & ICA & Yes & 250 & Decreased flow & 2 & $2 b$ & No \\
\hline
\end{tabular}

$\mathrm{M}$ indicates male; $\mathrm{F}$, female; $\mathrm{T}$, bifurcation of internal carotid artery; M1, pre-bifurcation segment of middle cerebral artery; M2, insular segment of middle cerebral artery; ICA, internal carotid artery; tPA, tissue plasminogen activator; SAH, subarachnoid hemorrhage; and *, repeated control angiograms showed maintained blood flow when the Solitaire stent deployed the occluded lesion after the third time. 
and the basilar artery $(\mathrm{n}=2)$.

An amount of $250 \mu \mathrm{g}$ of tirofiban was used in all patients except one $(500 \mu \mathrm{g})$. Repeated control angiograms after stent deployment showed maintained blood flow during 10 minutes in 8 lesions $(61.5 \%)$ (Fig. 1). Stent retrieval was required one time in 7 of these 8 lesions. Five lesions showed non-visualization or decreased blood flow in repeated angiogram, and 3 lesions ( 2 lesions; 2 times, 1 lesion; 3 times) required more than one attempt at stent retrieval in the 5 lesions. The overall frequency of Solitaire stent retrieval included one time in 9 lesions $(69.2 \%)$, two times in 2 lesions (15.4\%), and three times in 2 lesions (15.4\%). The occlusion sites requiring more than one attempt at
Solitaire stent retrieval were ICA $(\mathrm{n}=2)$, T-bifurcation of ICA ( $n=1)$, and the M1 segment of MCA $(n=1)$.

Successful recanalization was achieved for all lesions (TICI $3 ; n=8$, TICI $2 b ; n=5)$. Three procedural complications $(23.1 \%)$ developed, which included subarachnoid hemorrhage in 2 lesions (15.4\%) and hemorrhagic transformation in 1 lesion (7.7\%). In the current study, repeated angiograms showed that no vessel perforations or arterial dissections occurred during the procedure for patients with subarachnoid hemorrhage. Two patients with subarachnoid hemorrhage suffered no neurological deterioration after the procedure, and no concomitant parenchymal or intraventricular hemorrhage was observed. All 2 subarachnoid hemorrhages disappeared

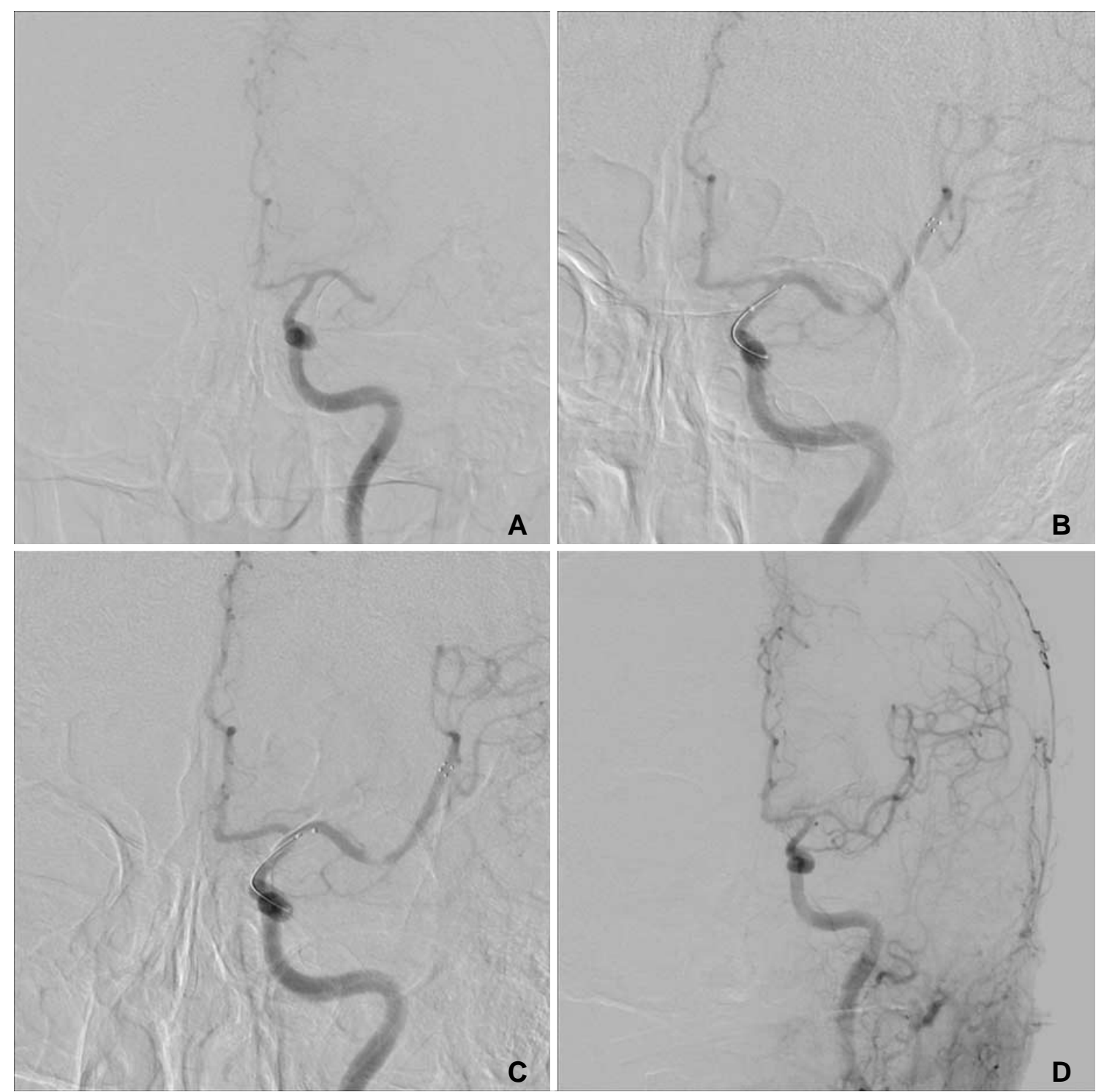

Fig. 1. Conventional angiograms from a 58-year-old man with a proximal middle cerebral artery occlusion (Case No.7).

A. Conventional angiogram shows left proximal middle cerebral artery occlusions. B and C. Serial repeated control angiogram showed no decrease in contrast filling of the occluded segment after solitaire stent deployment. D. Final control angiogram showed complete recanalization of the occluded segment after first retrieval of the solitaire stent. 


\section{Duck-Ho Goh, et al.}

on the 24-hour follow-up CT scan. One patient with an ICA occlusion beyond the time window of 8 hours showed one-fourth mismatched MCA territory on blood flow and blood volume maps of perfusion CT. This patient was expected to undergo decompressive craniectomy. Therefore, we decided to perform mechanical solitaire thrombectomy with low-dose booster tirofiban injection to have the possibility of avoiding decompressive craniectomy. Mechanical Solitaire thrombectomy with low-dose booster tirofiban injection resulted in near complete recanalization (TICI $2 \mathrm{~b}$ ), but hemorrhagic transformation (parenchymal hematoma, type 1) in the affected territory on brain CT was observed after the procedure. The patient underwent decompressive craniectomy to control increased intracranial pressure and remained in a severe disabled state 3 months after the procedure.

A total of 8 patients $(61.5 \%)$ recovered with good clinical outcomes $(\mathrm{mRS} \leq 2)$. Mortality occurred in the one patient with a basilar artery occlusion $(7.7 \%)$ due to reperfusion brain swelling and brainstem compression.

\section{DISCUSSIONS}

Because of the high recanalization rate and favorable clinical outcome, mechanical thrombectomy using stent devices has been regarded as the current standard treatment of acute ischemic stroke caused by embolic occlusion [1, 2]. However, because repeated control angiograms are not routinely performed after stent deployment, the conventional mechanical Solitaire thrombectomy method does not reveal the current status of the occluded lesion after stent deployment. In addition, the timing of stent retrieval varies according to the operators.

Repeated control angiograms will be informative as to the current status of the occluded lesion and the timing of the stent retrieval. If the repeated control angiogram shows no decrease or gradual increase of contrast filling in the stent deployed across the occluded lesion, the timing of solitaire stent retrieval might not be critical because blood flow will continuously supply the occluded lesion by the deployed stent and thrombolysis of endogenous tissue plasminogen activator will be enhanced through the patent lumen of the stent deployed across the occluded lesion. In the present study, repeated control angiograms revealed maintained blood flow in 8 lesions $(61.5 \%)$ and stent retrieval was required one time in 7 of these 8 patients. The patient with ICA occlusion required stent retrieval three times and showed maintained blood flow when the Solitaire stent was deployed to the occluded lesion after the third time. All lesions that showed maintained blood flow did not require additional stent retrieval. Maintained blood flow on repeated control angiograms suggested that recanalization would be high after stent retrieval.

If the repeated control angiogram showed non-visualization or decreased blood flow, the time delay of the stent retrieval might not be beneficial because thrombolysis of the endogenous tissue plasminogen activator would not be expected in the occluded lesion. In 3 of 5 lesions, non-visualization or decreased blood flow in repeated angiograms required more than one attempt at stent retrieval. Non-visualization or decreased blood flow on the repeated control angiogram might indicate a necessity of more than one time for stent retrieval, as the thrombus is difficult to withdraw as one piece without breakdown during stent retrieval.

A low-dose injection of tirofiban (up to $800 \mu \mathrm{g}$ ) showed no additive bleeding risk in the treatment of the occluded segment, according to our unpublished data and previous reports $[7,8]$. Alternatively, tirofiban infusion for the treatment of refractory occluded lesions showed favorable clinical and radiological outcomes [6,11]. Unlike the conventional Solitaire thrombectomy method, with no booster tirofiban injection, the low-dose booster tirofiban injection (up to $500 \mu \mathrm{g}$ ) might enhance the progression of thrombolysis and reduce the distal migration of the thrombus during solitaire stent retrieval, as tirofiban might resolve a secondary thrombus mainly composed of platelets resulting from the propagation of the primary thrombus in the initial occluded cerebral artery. Except for a small number of cases, the results obtained in the present study showed no distal migration of thrombus and successful recanalization of all lesions. These results might support the beneficial effects of low-dose booster tirofiban injection.

Selection bias was unavoidably included in the present study, as this study was a small-numbered retrospective analysis. However, the present study might not represent the easiness of thrombectomy because these studies included occlusion lesions of ICA or T-bifurcation in 5 lesions (38.5\%), which were difficult to recanalize after thrombolysis.

\section{CONCLUSIONS}

Our modified mechanical Solitaire thrombectomy 


\section{Mechanical Solitaire Thrombectomy with Low-Dose Booster Tirofiban Injection}

method using low-dose booster tirofiban injection might enhance the recanalization rate with no additive hemorrhagic complications.

\section{Acknowledgements}

This study was supported from the Korean Society of Interventional Neuroradiology in 2015.

\section{References}

1. Nogueira RG, Lutsep HL, Gupta R, Jovin TG, Albers GW, Walker GA, et al. Trevo versus merci retrievers for thrombectomy revascularisation of large vessel occlusions in acute ischaemic stroke (trevo 2): a randomised trial. Lancet 2012;380:1231-1240

2. Saver JL, Jahan R, Levy EI, Jovin TG, Baxter B, Nogueira RG, et al. Solitaire flow restoration device versus the merci retriever in patients with acute ischaemic stroke (swift): a randomised, parallel-group, non-inferiority trial. Lancet 2012;380:1241-1249

3. Miteff F, Faulder KC, Goh AC, Steinfort BS, Sue C, Harrington TJ. Mechanical thrombectomy with a self-expanding retrievable intracranial stent (solitaire ab): Experience in 26 patients with acute cerebral artery occlusion. AJNR Am J Neuroradiol 2011;32:1078-1081

4. Dorn F, Stehle S, Lockau H, Zimmer C, Liebig T. Endovascular treatment of acute intracerebral artery occlusions with the solitaire stent: Single-centre experience with 108 recanalization procedures. Cerebrovasc Dis 2012;34:70-77

5. Jeong HW, Jin SC. Intra-arterial infusion of a glycoprotein IIb/IIIa antagonist for the treatment of thromboembolism during coil embolization of intracranial aneurysm: a comparison of abciximab and tirofiban. AJNR Am J Neuroradiol 2013;34:1621-1625

6. Kwon JH, Shin SH, Weon YC, Hwang JC, Baik SK. Intra-arterial adjuvant tirofiban after unsuccessful intra-arterial thrombolysis of acute ischemic stroke: Preliminary experience in 16 patients. Neuroradiology 2011;53:779-785

7. Seitz RJ, Siebler M. Platelet GPIIb/IIIa receptor antagonists in human ischemic brain disease. Curr Vasc Pharmacol 2008;6:2936

8. Seitz RJ, Hamzavi M, Junghans U, Ringleb PA, Schranz C, Siebler M. Thrombolysis with recombinant tissue plasminogen activator and tirofiban in stroke: preliminary observations. Stroke 2003;34:1932-1935

9. Noser EA, Shaltoni HM, Hall CE, Alexandrov AV, Garami Z, Cacayorin ED, et al. Aggressive mechanical clot disruption: a safe adjunct to thrombolytic therapy in acute stroke? Stroke 2005;36:292-296

10. Berger C, Fiorelli M, Steiner T, Schabitz WR, Bozzao L, Bluhmki E, et al. Hemorrhagic transformation of ischemic brain tissue: asymptomatic or symptomatic? Stroke 2001;32:1330-1335

11. Baik SK, Oh SJ, Park KP, Lee JH. Intra-arterial tirofiban infusion for partial recanalization with stagnant flow in hyperacute cerebral ischemic stroke. Interv Neuroradiol 2011;17:442-451 